\title{
HISTÓRIA DA ELETRORRETINOGRAFIA
}

\section{The History of Electroretinography}

\author{
BACELLAR, M. ${ }^{1}$; MONTIANI-FERREIRA, F. ${ }^{2}$; SOMMA, A.T. ${ }^{3}$; BARROS FILHO, I.R. ${ }^{2}$ \\ ${ }^{1}$ Programa de Pós-Graduação em Ciências Veterinárias da UFPR \\ ${ }^{2}$ Departamento de Medicina Veterinária da UFPR \\ ${ }^{3}$ Pontifícia Universidade Católica do Paraná \\ Endereço para correspondência: Fabiano Montiani-Ferreira: montiani@ufpr.br
}

\section{RESUMO}

O olho juntamente com suas doenças vem sendo estudado desde o Egito antigo, sendo a oftalmologia, portanto, considerada como uma das primeiras especialidades médicas a existir. Com a invenção do oftalmoscópio no século XIX bem como de outros métodos de exames diagnósticos no século XX, iniciou-se a observação e o estudo da retina, tecido nervoso que realiza fototransdução e faz a passagem do estímulo elétrico para o cérebro, formando a imagem. A eletrorretinografia é um capítulo peculiar na história da oftalmologia. Trata-se de um exame oftálmico funcional que capta a resposta elétrica produzida pelas células da retina quando estimuladas. Tornou-se uma excelente ferramenta para estudar a funcionalidade da retina de animais e seres humanos, porém seu uso ainda encontra-se pouco difundido na Medicina Veterinária brasileira. $\mathrm{O}$ trabalho presente tem o objetivo de revisar a história deste valioso método de diagnóstico.

Palavras-chave: oftalmologia, Ragnar Granit, retina, eletrofisiologia, ERG.

\section{ABSTRACT}

The eye and its diseases have been studied since the time of the ancient Egypt; therefore, ophthalmology is recognized as one of the first medical specialties to ever exist. Along the years many advances in this area occurred, as the invention of the ophthalmoscope during the 19th Century and other ancillary methods in the 20th Century. After the invention of the ophthalmoscope, the observation and study of the retina, the anatomic part of the eye that transforms light energy into electric energy (phototransduction) stimulating the brain to form an image. Electroretinography is an ophthalmic examination that captures this electrical response generated by cells of the retina when stimulated by light. This is an excellent tool for studying the function of the retina, yet it is still a modestly widespread practice in the Brazilian veterinary medicine scenario. This paper aims to review the history of this valuable method of diagnosis.

Key words: ophthalmology, Ragnar Granit, retina, electrophysiology, ERG. 


\section{INTRODUÇÃO}

A retina é formada por várias camadas celulares, sendo estas responsáveis pelo fenômeno chamado de fototransdução, que é a transformação do estímulo luminoso em energia elétrica, estudado por fisiologistas e oftalmologistas (eletrofisiologia ocular).

A eletrorretinografia, como o próprio nome já diz, é um exame oftálmico que capta a resposta elétrica produzida pelas células da retina quando estimuladas pela luz. Trata-se de uma excelente ferramenta para estudar a função da retina, sendo um exame não invasivo e de fácil realização, permitindo assim um manejo seguro, mesmo nos casos em que se faz necessário a realização de anestesia (Heckenlively and Arden, 2006).

$\mathrm{Na}$ Medicina, a eletrofisiologia diagnóstica da visão pode ser estudada com 0 suporte dos exames de eletrorretinografia, eletrooculografia e o potencial visual evocado, importantes recursos na avaliação do nervo e vias ópticas, possibilitando o diagnostico das doenças hereditárias e das alterações isquêmicas da retina (Ávila, 2003).

$\mathrm{Na}$ Medicina Veterinária estes recursos e aparelhos são relativamente novos, ou pouco difundidos, necessitando de mais estudos e desenvolvimento de protocolos específicos às espécies domésticas. Este trabalho visa relatar a história da eletrorretinografia.

\section{DESENVOLVIMENTO}

A oftalmologia antes e após a invenção do oftalmoscópio

Em 1851, a invenção do oftalmoscópio monocular direto por Hermann von Helmholtz, hoje considerado pai da oftalmoscopia, ajudou na observação de problemas da retina por meio da observação do fundo ocular. Antes disto, na chamada era pré-oftalmoscópica, pouco se sabia a respeito da retina e suas funções. Foram feitos relatos de várias experiências sobre 0 fundo ocular, destacando-se a descrição de Jean Mèry, que em 1704, para estudar os movimentos da pupila, mergulhou um gato em um lago. Entrando em hipóxia, o olho do animal sofreu considerável dilatação pupilar. $O$ poder dióptrico da córnea foi então neutralizado pela presença da lâmina d'água, favorecendo assim a observação e descrição, pela primeira vez, dos vasos da retina (Ávila, 2003). Dez anos após a invenção do oftalmoscópio monocular direto Girard Teulon em 1861 introduziu o oftalmoscópio binocular indireto, que utilizava, por meio de espelhos, o reflexo da luz solar sobre a retina. Somente em 1885 foi introduzido o uso da luz elétrica na realização do exame (Ávila, 2003).

Novos métodos de exames foram sendo descobertos nos anos seguintes sendo que muitos deles, como gonioscopia, tonometria, eletrorretinografia e ultrassonografia ocular, são utilizados até hoje.

\section{A ELETRORRETINOGRAFIA}

Primeiros avanços no desenvolvimento do método

O alemão Emil Heinrich Du BoisReymond (1818-1896), considerado o fundador da eletrofisiologia moderna, nasceu na Prússia e viveu grande parte de sua vida em Berlim. Foi um dos fundadores da Sociedade de Física Alemã, sendo o sucessor de Johannes Muller como professor de Fisiologia na Universidade de Berlim, em 1858. Em seus trabalhos estudava as espécies de peixes capazes de produzir correntes elétricas. Foi 0 primeiro pesquisador a observar um potencial elétrico vindo do olho de um peixe em 1849, quando colocou um eletrodo na superfície da córnea (semelhante aos eletrodos usados hoje em dia). 
Já em 1865, o sueco Holmgren descobriu que existe resposta elétrica no olho após um estímulo luminoso, estudando sapos. Em 1880, retirando o segmento anterior do olho e colocando um eletrodo diretamente na superfície da retina, afirmou que esta estrutura de tecido nervoso era a responsável pela resposta elétrica gerada no olho (Rouck, 2006).

Na mesma época, outro pesquisador chamado Dewar relatou descobertas similares às de Holmgren. Ele mostrou que após estímulo luminoso incidindo sobre o eixo pupilar ocorria um pequeno movimento observado num galvanômetro, sugerindo que tal impulso elétrico positivo era produzido pela retina (Perlman, 2008). O galvanômetro é um instrumento que pode medir correntes elétricas de baixa intensidade, ou a diferença de potencial elétrico entre dois pontos.

\section{Primeiros eletrorretinogramas}

Em 1877, Dewar registrou o primeiro eletrorretinograma (ERG) no homem, mas não houve publicação do trabalho (Dantas, 1995). Gotch, em 1903, foi o primeiro a separar o resultado da ERG em duas ondas (Perlman, 2008). Em 1908, Einthoven e Jolly obtiveram excelentes dados detalhados do eletrorretinograma de um olho de sapo, utilizando novamente um galvanômetro. Foram os primeiros a classificar as diferentes porções do ERG usando letras: onda "a", primeiro segmento de onda negativo; a onda "b", segmento de onda grande positivo posterior a onda anterior; e onda "c", a outra porção positiva e um pouco menor (Einthoven and Jolly, 1908).

A primeira publicação de um ERG foi feita por Kahn e Lowenstein em 1924. A idéia era que o exame fizesse parte dos exames clínicos oftálmicos da rotina, mas os autores concluíram que existiam algumas dificuldades para execução do método. No mesmo período, Hartline desenvolveu outra maneira de colocar os eletrodos, aplicando um pedaço de algodão na superfície do olho para fixar o eletrodo e posicionando o eletrodo de referência na boca do paciente (Rouck, 2006).

Em 1929, Sachs mostrou que o ERG humano era dependente do sistema escotópico visual da retina. Em 1933, Cooper e associados acoplaram um amplificador no galvanômetro e obtiveram ondas com maior qualidade (Rouck, 2006).

\section{Ragnar Granit}

Ragnar Arthur Granit (1900-1991) foi um cientista que nasceu na Finlândia (Figura 1). Estudou medicina com ênfase na parte de psicologia. Durante seus estudos chegou à conclusão que a fisiologia seria melhor do que a psicologia para explicar o aparelho visual. Em 1926 aceitou o posto de assistente no Instituto de Fisiologia na Universidade de Helsinki, oferecido a ele pelo Professor Carl Tigerstedt. Granit formou-se em 1927, sendo convidado por vários institutos de pesquisa para trabalhar. Permaneceu meio ano em Oxford mudando-se na seqüência para a Pensilvânia. Voltou para a Finlândia em 1935 e assumiu a cadeira de professor de fisiologia. Durante a Guerra de inverno entre a Finlândia e a Rússia, Granit se refugiou na Suécia, permanecendo por lá como cientista.

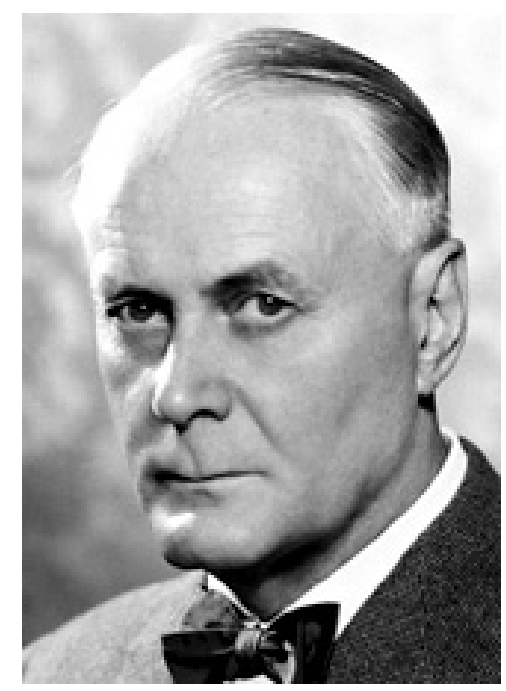

Fonte: Site Prêmio Nobel

Figura 1 - Ragnar Granit 
Ganhou inúmeros prêmios e, em 1967, junto com Haldan Keffer Hartline and George Wald, recebeu o prêmio Nobel em Medicina por seus estudos focados na eletrofisiologia.

Em 1933, utilizando gatos em estado de narcose induzida por éter e diferentes graus de oclusão da artéria carótida, conseguiu desdobrar os componentes do ERG (Porto, 2004). Observou a presença de três potenciais, os quais nomeou: PI, PII e PIII, de acordo com o desaparecimento deles durante a anestesia e hipóxia. O componente corneal negativo PIII correspondia à onda "a"; o potencial corneal PII, que era inclusive maior e dependente do primeiro, correspondia à onda "b"; e quando PII diminuía, formavase de forma devagar o componente $\mathrm{PI}$ correspondente à onda "c" (Rouck, 2006).

No ano de 1935, Granit estudou o ERG de diferentes animais e dividiu em dois tipos de retina: retina do tipo $E$ (excitatória) e retina do tipo I (inibitória). No tipo $E$, predominam as respostas originadas dos bastonetes, revelam uma ampla onda "b" ao estímulo luminoso, tendo como exemplo a retina humana; e no tipo I, as respostas dos cones, com a predominância do traçado negativo (onda "a"), retina de pombo (Dantas, 1995).

No passar dos anos, mais estudos sobre os componentes do ERG ocorreram e pôde ser observada a relação das porções da retina com as ondas do ERG. Noell (1952) por meio de um estudo, concluiu que o epitélio pigmentado da retina (EPR) que produzia a onda "c" e o potencial transretiniano (Rouck, 2006), neste mesmo ano, alguns trabalhos, Armington, Jonhson e Riggs, assim como em 1945, Adrian relatou, confirmaram a existência de componentes fotópicos (difásicos, com presença de uma onda negativa seguida de uma positiva) produzidos por luz branca ou qualquer luz monocromática, exceto a azul e componentes escotópicos, monofásicos, produzidos por todas as cores exceto a vermelha (Dantas, 1995).

Outro estudo demonstrou que a onda "b" é gerada pela camada de células que se encontra entre a membrana limitante interna e a externa. Esta descoberta foi detalhada primeiramente em 1969 por Faber. Steinberg e colegas (1970) investigaram as respostas produzidas pelo EPR e provaram que a origem da onda "c" é devido a uma redução na concentração de íons de potássio no espaço subretiniano, causando polarização da EPR (Rouck, 2006).

\section{ERG hoje}

Por meio de estudos e do melhor entendimento sobre os componentes do $E R G$, este exame foi aperfeiçoado e introduzido na rotina da oftalmologia clínica. Com o tempo tornou-se o método diagnóstico mais preciso para a avaliação da retina (Komaromy et al, 1998), porém não pode assegurar que 0 paciente realmente tenha visão.

$\mathrm{Na}$ Medicina, a ERG é usada tanto para diagnosticar como para avaliar a progressão de degenerações da retina, intoxicações e, no caso de pacientes diabéticos, para diagnosticar e acompanhar a retinopatia proliferativa e não proliferativa (Ofri et al, 1993). Na Veterinária, além de servir para diagnóstico de várias retinopatias e como exame pré-cirúrgico para a realização da cirurgia de correção de catarata nas diferentes espécies de animais (Figura 2), o exame serve como importante modelo biológico para estudo das degenerações de retina que acometem o homem (Petersen-Jones et al, 2006). Com o avanço da tecnologia, particularmente dos microprocessadores e diminuição do tamanho dos amplificadores de sinal elétrico os modelos foram se tornando cada vez mais complexos e compactos.

Segundo Miyake (2008), o eletrorretinógrafo é formado pelos eletrodos, a fonte de estímulo luminoso e o 
aparelho que capta e registra o estímulo. São três eletrodos principais: o eletrodo que capta a resposta ("recording electrode"), o eletrodo referência ("reference electrode") e o eletrodo "terra" ("ground electrode"). O primeiro fica localizado em contato com a córnea ou próximo à conjuntiva bulbar, sendo que o modelo mais utilizado é a lente de contato que possui um círculo de ouro para a condução do estímulo (ERG-jet lens). O eletrodo referência pode estar incorporado a lente de contato (no caso dos eletrodos bipolares) ou não (no caso dos eletrodos monopolares). Quando sozinho, deve estar localizado próximo a órbita, na região temporal, cerca de 3 a $5 \mathrm{~cm}$ do canto temporal do olho sendo examinado
(Mentzer et al., 2005). O eletrodo "terra" pode estar localizado em qualquer parte do corpo, sendo que normalmente é posicionado na orelha ou na região occipital.

O traçado do eletrorretinograma é composto por três ondas básicas (Figura 3). A onda "a" (onda inicial negativa), formada pelas correntes iônicas extracelulares geradas pelos fotorreceptores (cones e bastonetes). A onda "b" (onda positiva maior mais lenta) reflete principalmente a atividade das células bipolares da retina, que se encontram entre os fotorreceptores e as células ganglionares, transmitindo os sinais dos fotorreceptores para as células ganglionares (Heckenlively e Arden, 2006).

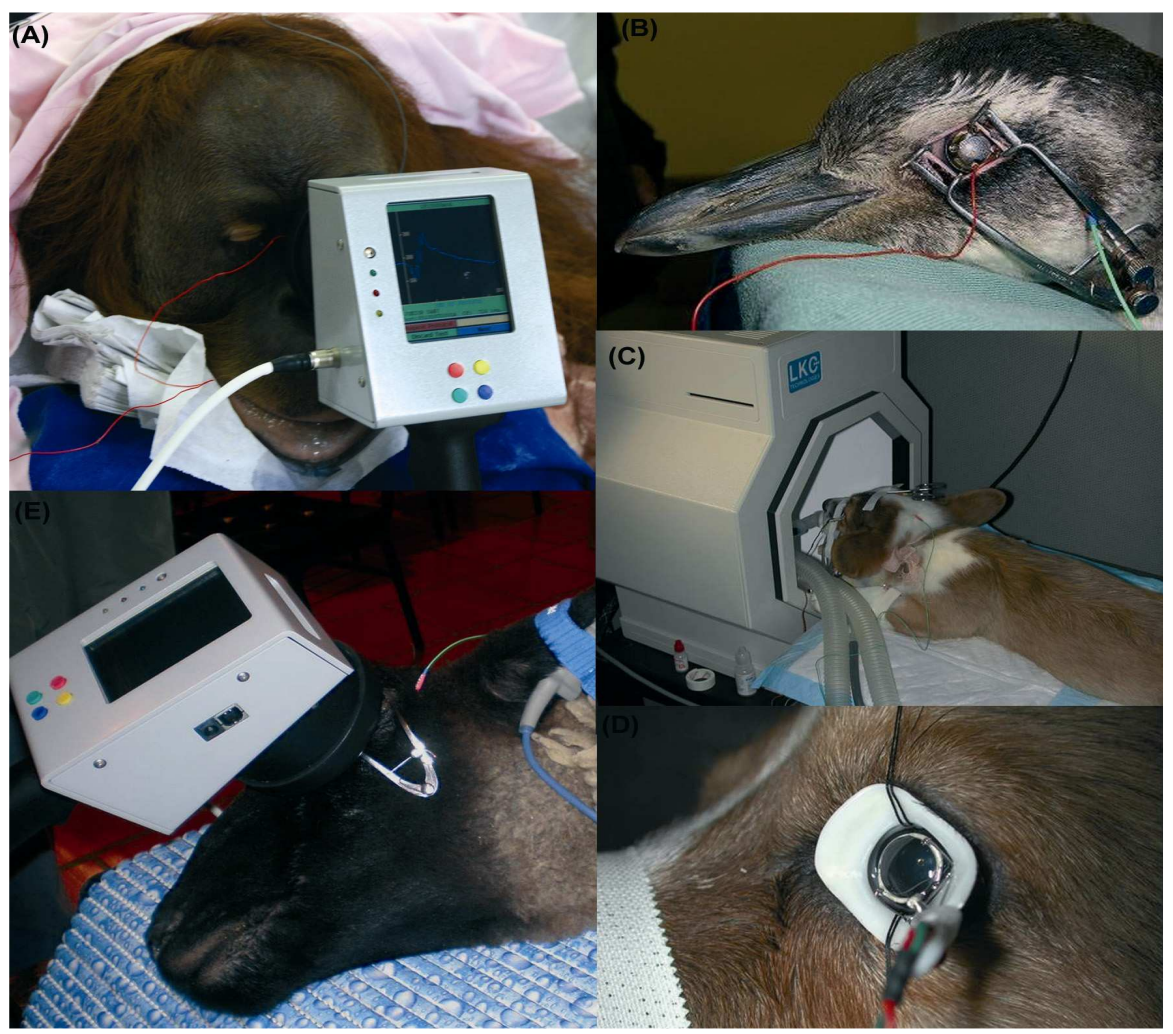

Figura 2- Exames eletrorretinográficos em várias espécies de animais. (A) Exame sendo realizado em um exemplar de orangotango (Pongo pigmeus) empregando o aparelho de ERG HMsERG da marca RetVet (Columbia, MO); (B) Detalhe da localização do eletrodo monopolar do tipo "ERG-jet lens" e do eletrodo referência (próximo ao blefarostato) durante o exame em um exemplar de Pingüim-de-Magalhães (Spheniscus magellanicus); (C) Detalhe de um estimulador de campo inteiro (Ganzfeld) da marca LKC (Gaithersburg, EUA) durante o exame em um cão (Canis familiaris) da raça Cardigan Welsh Corgi; (D) Detalhe da localização do eletrodo bipolar do tipo Burian-Allen no mesmo cão. Note os fios de sutura fixando o eletrodo na posição correta. (E) Ovelha (Ovis aries) da raça Suffolk durante exame empregando um HMsERG (RetVet Corp., Columbia, MO). 
A onda "c" (onda positiva de aparecimento bem mais tardio) está relacionada com a polarização do epitélio pigmentar da retina (EPR) (Gum, 1980) e pode ser observada quando é feito um tipo especial de ERG, o ERG de longos flashes. Enquanto as ondas "a" e "b" aparecem na escala de milisegundos, a onda "c" aparece na escala de segundos. O aparecimento da onda "c" no epitélio pigmentar da retina depende do fluxo de potássio das outras camadas da retina. Só que o fluxo extracelular de potássio depende da fototransdução. Portanto, a onda "c" pode nos dar um panorama da fototransdução como um todo, da integridade do epitélio pigmentar da retina e da interação do EPR e do resto da retina. A pesquisa da onda "c" não é rotineira, geralmente só é pesquisada quando já se sabe que há algo errado com o ERG de flash-padrão e, conseqüentemente, com a retina.

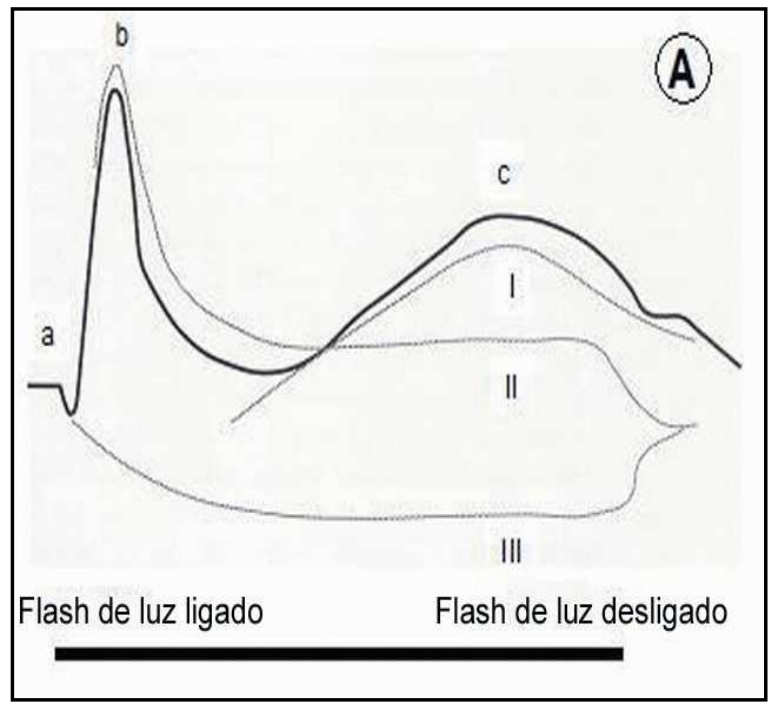

Figura 3 - Traçado esquemático de eletrorretinograma. (A) potenciais de ação, ou componentes PI, PII e PIII.

\section{CONSIDERAÇÕES FINAIS}

Na Medicina Veterinária, o ERG é utilizado principalmente como exame pré-cirúrgico para a avaliação da retina em pacientes com catarata. $\mathrm{Na}$ oftalmologia de pequenos animais, também é útil para classificar e diagnosticar precocemente a atrofia progressiva de retina (PRA) (Figura 4), além de diferenciar a degeneração súbita adquirida de retina (SARD) da neurite óptica (Liapis, 2004).
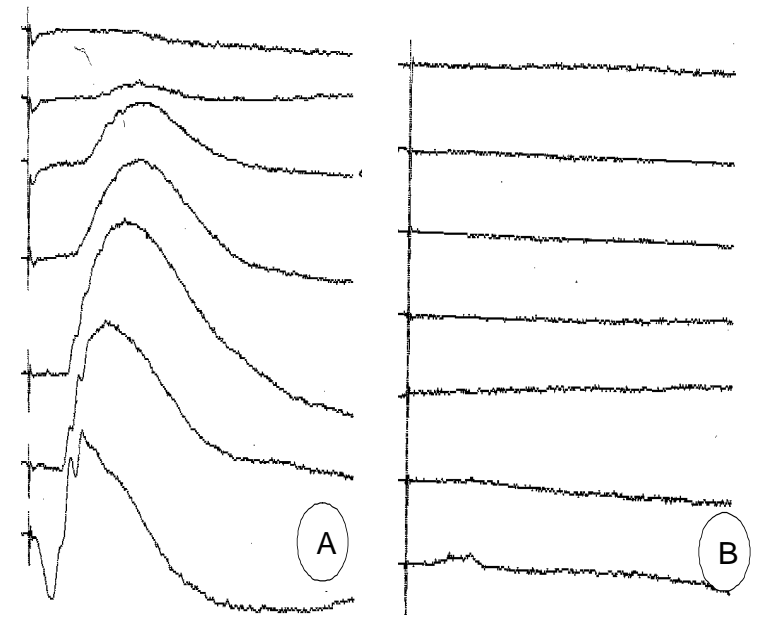

Figura 4. Traçados reais de eletrorretinograma dispostos como respostas a uma série de flashes de intensidade crescente. As respostas para os flashes de menores intensidades estão mais acima, passando para os de maior intensidade abaixo da figura. A) Note a resposta normal de um cão adulto da raça Cardigan Welsh Corgi. B) Série de respostas em um cão de mesma idade e raça sofrendo de atrofia de retina progressiva. Note o aspecto achatado, sem ondas "a" ou "b".

No Brasil, o uso do eletrorretinógrafo na Medicina Veterinária ainda é recente, mas em amplo crescimento, visando principalmente 0 estudo das doenças da retina, vislumbrando ainda o estudo comparado entre animais e o homem.

\section{REFERÊNCIAS}

ÁVILA, M. A Retina no Século XXI. In: XXXII Congresso Brasileiro de Oftalmologia. 2003, Salvador, BA.

Biografia Ragnar Arthur Granit. Nobel Prize. Disponível em: 
http://nobelprize.org/nobel prizes/medicine/laurea tes/1967/granit-bio.html. Acesso em: 01/09/2008.

Biografia Emil Heinrich Du Bois Reymond. Enciclopédia Britannica Online. Disponível em: http://www.britannica.com/EBchecked/topic/43006 7/ophthalmology. Acesso em: 01/09/2008.

DANTAS, A.M. Eletrorretinografia. In: DANTAS,A.M; COSTA, J.G.C.; NETO, L.P; YAMANE,R.; ELIAS,C.A. Eletrofisiologia Ocular.1.ed. Rio de Janeiro: Editora Cultura Médica, 1995. p.141-225.

EINTHOVEN, W.; JOLLY, W.A. Form And Magnitude Of The Electrical Response Of The Eye To Stimulation By Light At Various Intensities. Quarterly Journal of Experimental Physiology, n. 1, p.373-416, 1908.

GUM, G.G. Electrophysiology in veterinary ophthalmology. Veterinary Clinics of North America: Small Animal Practice, v.10, p.437454, 1980.

\begin{tabular}{|c|c|c|}
\hline ENLIVELY, & J.R. and & \\
\hline $\begin{array}{l}\text { Principles and } \\
\text { Electrophysiology } \\
\text { Massachusetts: MIT }\end{array}$ & $\begin{array}{c}\text { Practice } \\
\text { of } \\
2006\end{array}$ & $\begin{array}{c}\text { of } \\
\text { Vision. }\end{array}$ \\
\hline
\end{tabular}

KOMAROMY, A.M.; SMITH, P.J.; BROOKS, D.E. Electroretinography in dogs and cats. Part I. Retinal morphology and physiology. The Compendium on Continuing Education, v.20, p.343-354, 1998a.

KOMAROMY, A.M.; SMITH, P.J.; BROOKS, D.E. Electroretinography in dogs and cats. Part II. Technique, interpretation and indications. The Compendium on Continuing Education, v.20, p.355-366, 1998b.

LIAPIS,I.C. Electroretinography in Small Animal Practice. In: 29th World Congress of the World Small Animal Veterinary Association. 2004, Rhodes, Greece.

MENTZER, A. ; EIFLER, D. ; MONTIANIFERREIRA, F. ; TUNTIVANICH, N. ; FORCIER, J.; PETERSEN-JONES, S. Influence Of
Recording Electrode Type And Reference Electrode Position On The Canine Electroretinogram (erg). Documenta Ophthalmologica, v. 111, n. 111, p. 95-106, 2005.

MIYAKE, Y.; BRIGELL, M.; FULTON, A.; HOLDER, G. E.; MARMOR, M. Standard for clinical electroretinography (2008 update). In: The ISCEV XLVI Annual Symposium. 2008, Morgantown, USA.

OFRI,R; DAWSON,W.W; FOLI,K; GELLAT,K.N. Chronic ocular hypertension alters local retinal responsiveness. British Journal of Ophthalmology, v. 77, p. 502-508, 1993

PERLMAN, I. The Electroretinogram: ERG. Webvision. 2008. Disponível em: http://webvision.med.utah.edu/ERG.html. Acesso em: 04/09/2008.

PETERSEN-JONES,S.; TUNTIVANICH, N.; MONTIANI-FERREIRA, F.; KHAN,N. Electroretinograms of dog and chicken. In: HECKENLIVELY,J.R and ARDEN,G.B. Principles and Practice of Clinical Electrophysiology of Vision. 2.ed. Massachusetts: MIT, 2006, p.911-921.

PORTO,F.B.O. Eletrorretinografia Multifocal na Retinose Pigmentaria. Belo Horizonte, 2004.174p. Dissertação (Doutorado em Medicina), Curso de Medicina, Universidade Federal de Minas Gerais.

ROUCK, A.F. History of Electroretinogram. In: HECKENLIVELY,J.R and ARDEN,G.B. Principles and Practice of Clinical Electrophysiology of Vision. 2.ed. Massachusetts: MIT, 2006, p.3-10.

TROMPOUKIS,C; KOURKOUTAS,D. Greek mythology: the eye, ophthalmology, eye disease and blindness. Canadian Journal of Ophthalmology, v.42, p. 455-459, 2007.

WHEELER, J.R. History of ophthalmology through the ages. British Journal of Ophthalmology, n.30(5), p. 264-275, 1946. 